\title{
Mobile Animation Algorithm for Cyber Museum
}

\author{
Sung-Soo Hong ${ }^{1}$ and Sang-Kil Kim ${ }^{2}$ \\ 1 Dept. of Computer Eng. Hoseo University, Korea \\ sshong@office.hoseo.ac.kr \\ 2 Dept. of Computer Eng. Hoseo University, Korea \\ ksknet@empal.com
}

\begin{abstract}
Communication technologies have been brought tremendous change to various aspects of an ever-fast changing world at present. Particularly, the use of Internet and cyberspace is widespread in every corner of our life. In this paper we propose a gesture algorithm using an animation technology. It was developed for educational purposes, and accessible through the WWW of Internet. The visual processes can be adapted for a variety of fields. For instance, in Cyber Clam Museum, the visual processes were used to design a screen with a realistic image and create a system that makes possible show at 360. Therefore, in the system, mobile hosts can observe the detailed clam's structure and save the image. This proposed algorithm provides multiple techniques to user may manipulate, visualize and interact with image on the Web. And every such transformation as translation, rotation, and scaling can be applied in the picture interactively for the convenient and effective viewing. ...
\end{abstract}

\section{Introduction}

As information industry has been developed modern times, the demand for useroriented information has increased more than text-oriented one. Human being prefer visual technique method to written text, for the visual recognition has been developed in recent years. Especially, he or she is trying to make every effort to communicate more easily with information users with visual configuration, using computer and through them. [3]

The previous computers provided only linguistic and two-dimensional pictures and to and to use mouse and keyboard only to control cyber world. But, it didn't provide enough for information users to believe the world as he or she saw it in cyber world. Displaying information on Internet gives users both timesaving and economic benefits. To provide museum and other useful information on Internet or through Internet will give much more educational effects. One of new methods which constructed museum on Internet, especially visualization, is new one which is generating interaction between computer and human. This visual scheme is creative art in expressing graphic and factual image, depicting on screen and giving the feeling of real thing vividly. Of course in mobilizing human imagination through three-dimensional space it is useful too. There are 
a lot of problems to solve in order to construct factual image on Internet. First, the biggest problems of all, is the lacking of reality and control of the image for users. Furthermore, there is a problem in terms of Internet transmission velocity. In order to transform a real thing into object in cyber space through computer, the art which deals with factual method with three-dimensional space is required. That is, the emphasis on visualization make us artificial feeling without understanding traits of real objects. On the contrary, the constructing on factual movement causes this disharmony, confusion between fact and visual situation. The visualization using the JAVA can solve these problems at once. The animation generating from it can express real things freely and make the control of image on screen smoothly according to intention of producer. We can display animation on screen, stop, enlarge, contract, and control animation speed freely. Thus we can construct user-oriented system.

\section{Related Work}

One of the goals of image-based animations is rendering. There have been many great minds in the field. Chen [11] created images at a new point of view by preserving the images from one frame to another instead of using the traditional 3D rendering. Expanding [11] and Chen [12] introduced the Quick Time VR, which provides many different points of view using the environmental map from the real world to create images. In making panoramic images, Szeliski [4] used a new technique, which has no limits in inputting images. This rotating matrix technique only requires three parameters instead of typical eight and thus lets the user make panoramic images in a fast and easy way. Solving the problem of unexpected curves with the image technique Chen used in View Interpolation, Seitz [4] applied the principles of the projective geometry and called his way View Morphing. Levoy [8] interpreted that the 2D slices in a $4 \mathrm{D}$ function made a set using new images and created images in new points of view by sampling with no 3D information such as particular point matching. Horry [14] suggested Tour into the Picture, with which you can do animations by only using a piece of $2 \mathrm{D}$ image. Although it's amazing in that you can create animations even without a perfect 3D environment, it does have a disadvantage that you should make distinctions of backgrounds and select vanishing points manually. In his new portal texture algorithm, Aliage[1] divided the inside of a building into two groups, one of which is the space surrounded by the walls and the other of which is the portals that can been in the space such as doors, windows, and furniture. Then he replaced the geometric models seen through the portals with images. While the old approach presented all 3D models seen through the portals, this new method provides $2 \mathrm{D}$ images, which makes real-time processing possible. Debevec and Niamark succeeded in making the virtual environment of a Canadian national park by installing two $16 \mathrm{~mm}$ cameras and catching the stereo images every meter. Kanade[13] introduced a stereo image matching technique with 50 cameras. Debevec[9] used aerial photographs to suggest a new approach to modeling and rendering a building. In this approach, the prepared 3D structures will 
come up as the user moves the pre-defined primitives over the pictures and gets close to a boundary.

Recently, the technology for providing three-dimensional image on Internet has been regarded as an important field. Indeed, VRML that makes threedimensional graphic image, requires VRML Plug-in set-up and is difficult to express a real image. Also, users should take a special training to operate the system. Moreover, the translating rate becomes much slower with a big file [3][5].

Currently, information through the Internet is provided by means of the first dimensional image or text. Although there are several ways to transfer a variety of information, it is rare to be shown as three-dimensional translation on the web. Such a three dimensional presentation is normally carried out in the space through Java Applets program. In fact, the JAVA language may be the best tool for expressing a three dimensional image on the Internet. However, there are still some problems such as ways to add real images to the Internet graphic or the object in the three dimensional space. The three-dimensional graphic tool or image-extracting procedure may be available to visualize the object. However, three-dimensional images can be created on the Internet by producing an optical illusion through a succeeding two-dimensional image. Indeed a system using a realistic image is a better way rather than simple graphic manufacturing for the creation of an effective visualization [10].

\section{Mobile Animation Model}

In a theoretical point, it is natural to make an imaginary actuality equivalent to real condition by using of imagination. On the other hand, an imaginary actuality may become a new art mode in a point of artistic view. As artists create a world through a painting, a musical score, a stage or a stone, the world of imaginary actuality will be artificially realized in the computer. If users plunge into the imaginary actuality and interact with a variety of items in the world, they must obtain a better pleasure. Now we first suggest a model for the interaction between users and system for the animation of imaginary actuality. How can we define a relationship between users and system for the animation of imaginary actuality? And what is the basic relationship? A model that we are to suggest in the present paper is based on the model provided by J. Latta et al. [2]. Our model mainly consists of inter-phases connecting human with imaginary environment. The final goal of our animation system of imaginary reality is that when users replace a real situation with imaginary one, users cannot realize any difference between two situations. Furthermore, our system shall enable users to feel the better color sense or contours.

As shown in Figure 1, users can look real things through a monitor on displaying module. Sensing module in our system is to sense human's action or gesture. This module includes a variety of sensors and digital camera equipped with input and output devices. Information received through above two modules is processed on Virtual Perception module and extracted according to user's idea. This module connects the physical module with the logical sensor and a 


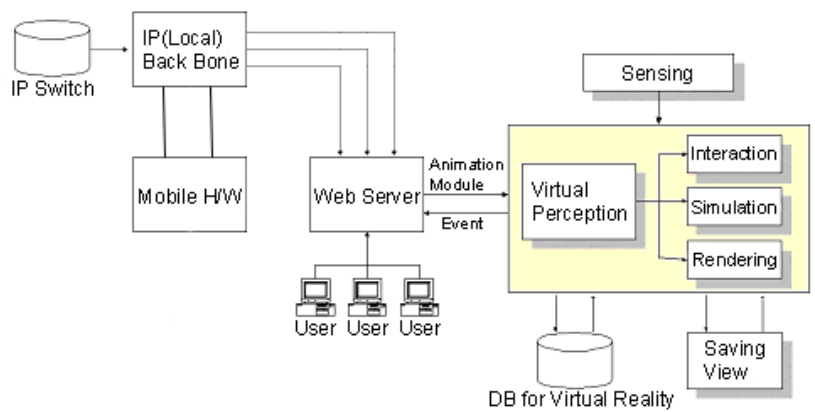

Fig. 1. Virtual Reality Animation Model

limitation that can be interacted with imaginary world is determined according to user's goal. All these works are determined on Interactive module.

Interactions with pre-determined environment are actually performed in the Simulation module. Of course, users can operate the basic processes without establishment of Interactive. Rendering module takes a part to draw the changed imaginary world. This module can reduce or magnify a real object and make a rotation to all sides in the monitor. The DB for the imaginary reality contains an image and a text of all objects in the imaginary world. This also includes geometrical, physical and behavioral characteristics on the intellectual action. Saving view can save and output the part that a user aims to.

The animation algorithm consists of an Init processor, a Display processor, a Zoom processor, and a Speed processor and all processors run by calling a run processor. The animation is done by creating a file from the image to be animated taken by a camera and by utilizing a Java program that is saved in the memory address of an image. In the run processor, a thread runs first to determine a rotation/stop and left/right rotation situation and then the animation is performed sequentially if the satisfaction is made. The reverse operation is performed in the case that the nix is reducing. Then, the Speed processor and Display processor are called.

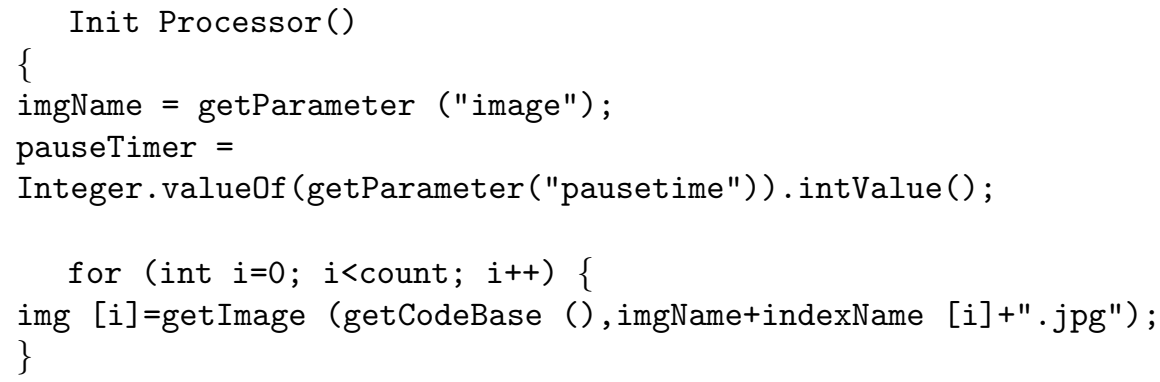




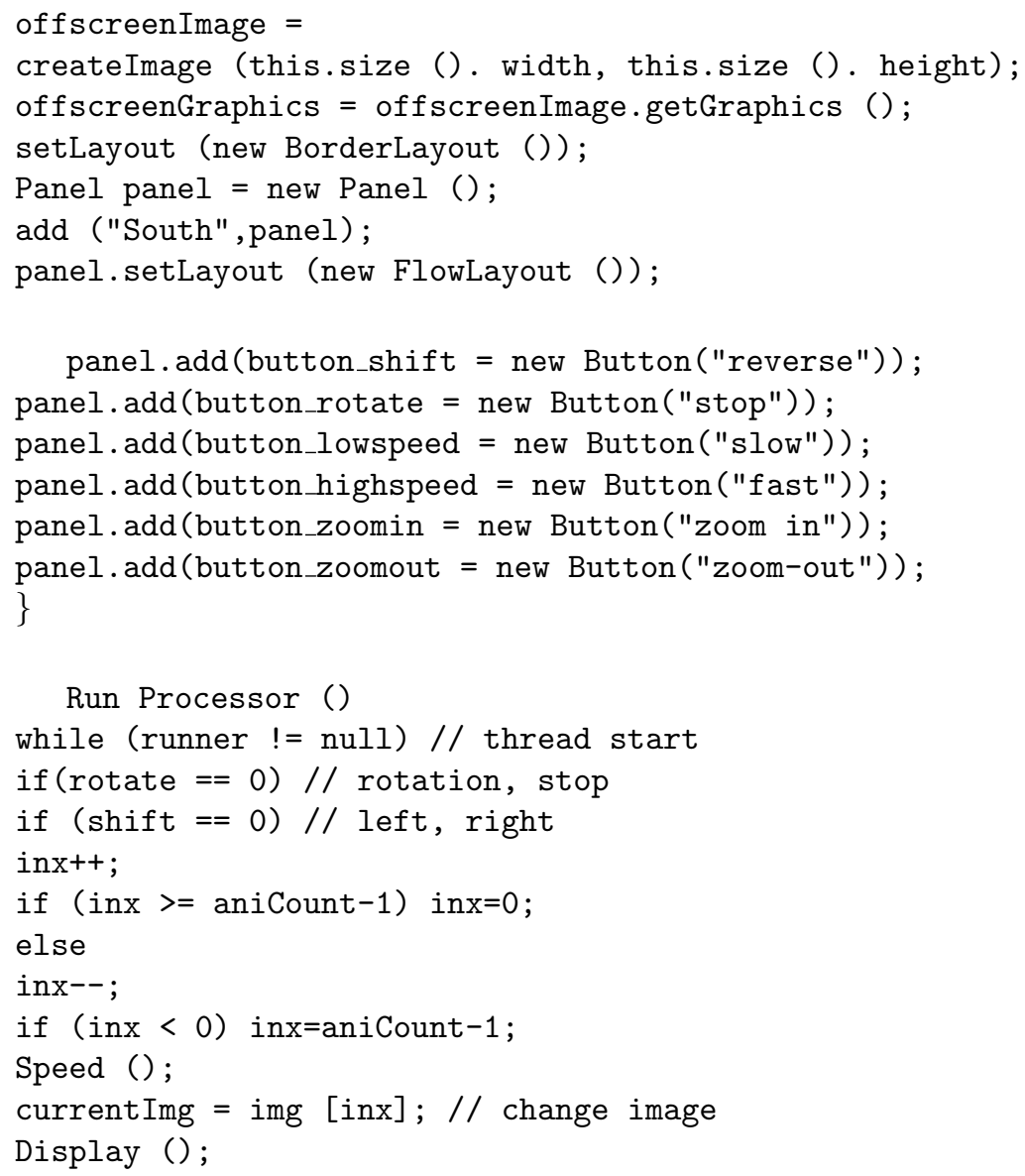

The display processor is one that displays an image sequentially on the screen after getting the information of the size and position of an image to be displayed by calling a zoom processor. The xpos represents an x-axis position, ypos is a y-axis position. Img Width is the width of an image, imgHeight is the height of an image, and drawImage is a Java function that draws an image.

\section{Display Processor ()}

\{

Zoom (); // Calling zoom processor

int imgWidth = currentImg.getWidth (this);

int imgHeight $=$ currentImg.getHeight (this);

if $(\mathrm{zoom}==1)\{$

xpos $=0$;

ypos=0; 


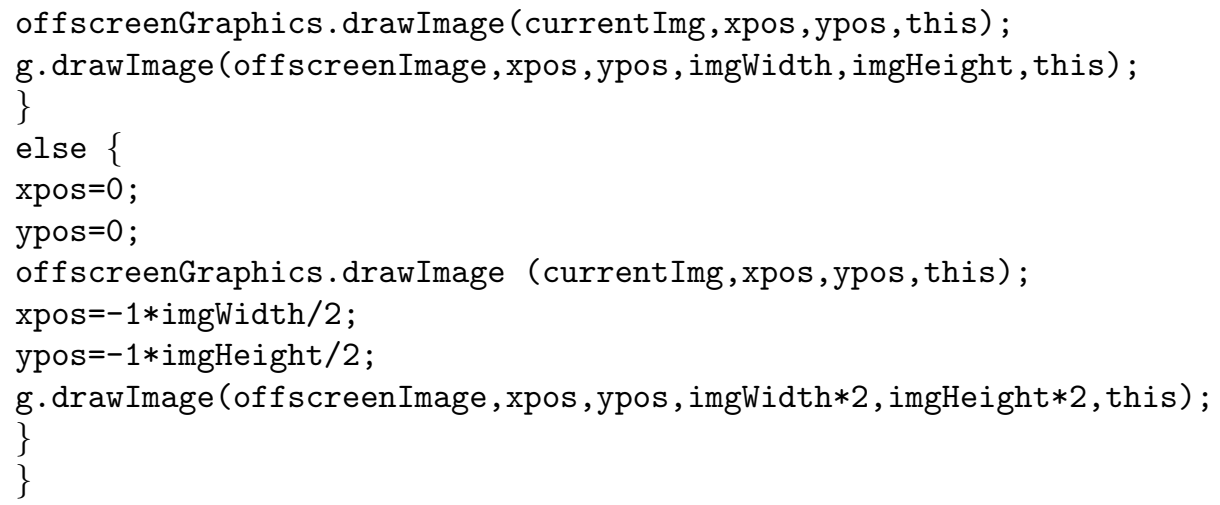

The zoom processor is one that controls the size of an object. Here, the current image is the image to be displayed, the zoom parameter controls the image with the step of $1 / 10$, and xpos and yos are an $\mathrm{x}$-axis and $\mathrm{y}$-axis positions of an image to be displayed, respectively.

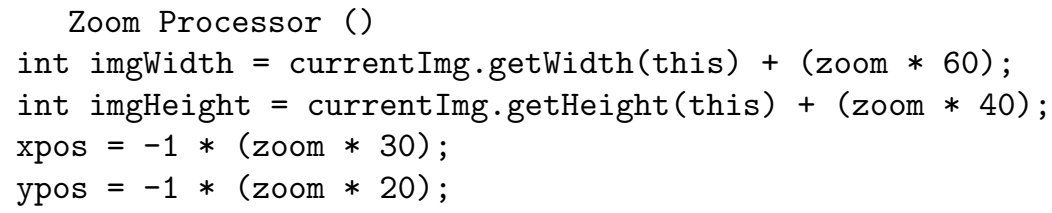

The speed processor is one that makes the speed of an image to be animated as optimum as possible.

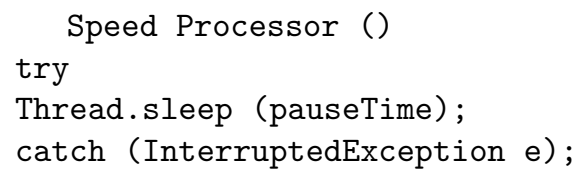

\section{Implementation}

The Cyber Shell Museum is implemented using HTML, Java Script and Java based on the Web. Technologies employed in this paper can provide better reality than still images of existing museums through visual effects that allows zoom, rotation as well as still views as shown in http://cyber.hoseo.ac.kr. The implementation has performed as follows: First, digital camera digitizes real shells with 360 rotational views or using animation technologies on developer's purpose. Second, real parts of shells are extracted and their sizes are adjusted to 
employ using the Photoshop. Third, animation programs were developed to provide interactions with users considering zoom-in, zoom-out, 360-degree rotation. Finally, background of shells was determined and Java programs were developed to get system worked as animation.

For the shells of the world, about 1,000 species of shells are selected and they are classified into 5 kinds; Gastropods, Bivalves, Tusk shells, Chitons and Cephaloplds, and more detailed classification has been done respectively. For example, in case of Gastropods, shells are classified into nine kinds of Ear, Cap, Pear, Corkscrew, Top, Spindle, Club, Barrel, and Egg using the appearance of shells in Fig. 2.

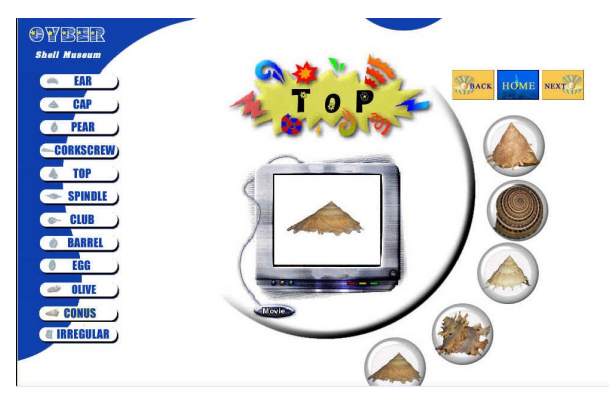

Fig. 2. World Shell

The Bivalves is classified into 7 categories, i.e. Discus, Fan, triangular, Boat, Paddle, Heart, Irregular. If the CLUB of Gastropods is clicked using mouse, selected kinds of shells are displayed in the left side of the screen. A user can select one of them to see related information and the front view of the selected shell through animated pictures. With various functions such as still picture, zoom-in, zoom-out, 360-degree rotation, the use can enjoy nice virtual reality in Fig. 3.

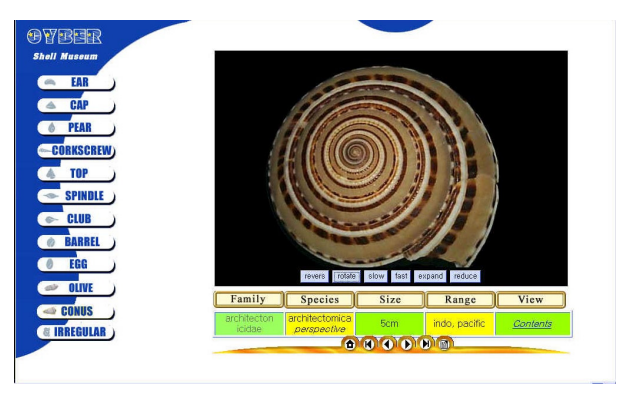

Fig. 3. Virtual Reality of Shell 


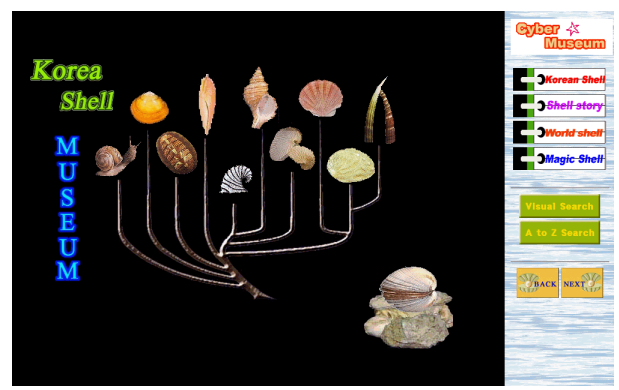

Fig. 4. Korean Shell

For the shells of Korea in Fig.4, shells are classified into 10 categories and selected shells are displayed continuously with time interval. The world of Marvelous-shells contains 20 kinds of marvelous shells in the world and gives uses curiosity due to the fancy looking shells.

The Story-shells was implemented in order for students to get knowledge about shells. For example, it provides pictorial and textual information for easy understanding about what Gastropods is, terminologies for spire, aperture, apex, growth line, and etc. The Rare-shells contains five categories about rare shells in the world. A user can search shells by using images when the user doesn't know shells' name or appearance in Fig. 5.

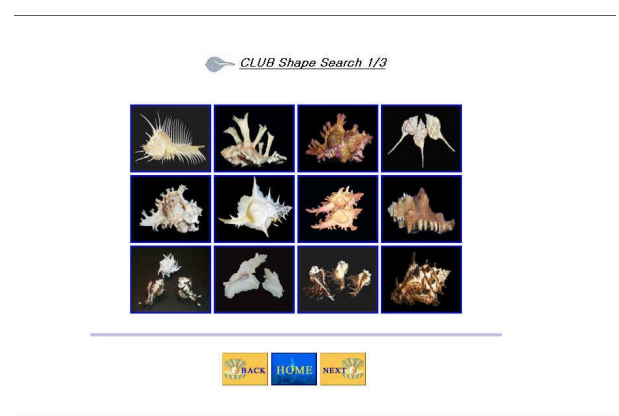

Fig. 5. Visual Search

If the user knows shell's name, the textual search can be used with shell's names and characteristics in Fig. 6.

After experiment was done by joining the shell museum system on Internet network (http://cyber.hoseo.ac.kr), advantages were found. The differences and advantages of using the animation technology with VRML system [Table. 1]. 


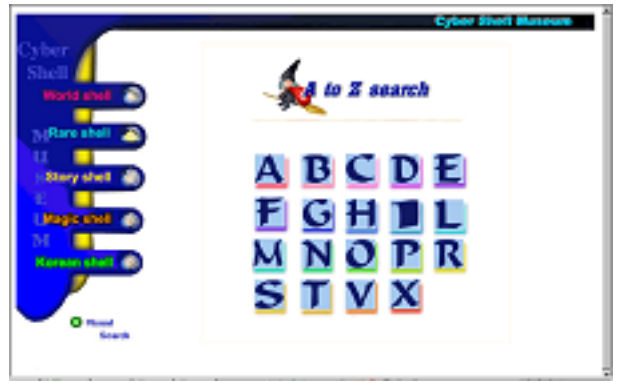

Fig. 6. Textual Search

Table 1. Comparison of Animation technology and VRML

\begin{tabular}{lll}
\hline & Animation & VRML \\
\hline Advantage & factual mage & cyber image \\
Need System & No & Plug-in program \\
Image express & very easy & very hard \\
Dimension & $3 D, 2 D$ & $3 D$ \\
Train & No & special train \\
Memory & small file & big file \\
Internet & easy & very difficult \\
Speed & fast & slow \\
\hline
\end{tabular}

In deed, VRML system that makes three dimensional graphic image, but there are big problem. First VRML system need a plug-in setup program. Second it is very difficult to express a real image. Also, users should take a special training to operate the system. Moreover, the translating rate becomes much slower with a very big file. So, we can not accept mobile system. The animation system can express real images freely and make the control of image on screen smoothly according to intention of Producer. We can display animation on screen, stop, enlarge, contract and continue animation speed freely. Thus we can construct animation system based on the mobile system.

\section{Conclusion}

In this paper we showed the similar system three dimension, using shell museum on cyber space technique and animation. So far we have utilized simple image, and the graphic visual objectives on Internet. Besides these, we could give more 
detailed information that of going to museum in person using the factual image. The visualization of utilizing Java makes the Internet users who are familiar with complicated and difficult information understand easier. Furthermore, the users control the necessary information which is available directly. What is called a kind of users-oriented system? The weak-points of this system realized rather slow when we use telephone line. But we can solve the problem naturally by improving Internet transmission velocity promptly and the processing speed of computer. This paper, different from the visual image of graphic, gives not only users using the Internet much interests but also, the educational value. This cyber shell museum provides new conceptual Internet information and share materials which are display on web beyond space limitation. So far, we construct based on the materials for shell over 1000 species worldwide with animation.

\section{References}

1. D.G. Aliage, Anselmo, A. Lastra, "Architectural Walkthroughs Using Portal Textures", IEEE Visualization97, pp. 355-362,1997.

2. J. Latta, D. Orberg, A conceptual Virtual Reality Model, IEEE Computer Graphic and Application; vol. 4, No.1, pp. 23-20, Jan, 1994

3. John J. McArdle, "Benefits and Limitation of Mega-Analysis Illustrated using the WAIS, Vol.18, No.1, pp. 12-16 Sep, 2002

4. J.W. Shade, S.J.Gortler, R.Szeliski, "Layered Derpth Images" , SIGGRAPH98, 1998.

5. Kah-Seng Chung, Yia fourg Chen, "A versatile Digital Mobile Channel Simulation." Apcc 2002, pp. 10-14, Sep. 17. 2002.

6. L. McMillan, G. Bishop, "Plenoptic Modeling: An Image-Based Rendering System ", SIGGRAPH95, pp. 39-46, 1995.

7. M. Brady et al, VRML Testing: Making VRML Worlds Look the same Everywhere, IEEE Computer Graphics and Application, Mar 1999, pp. 59-67

8. M. Levoy, P. Hanrahan, "Light Field Rendering", SIGGRAPH96, pp. 31-42, 1996.

9. P. Debvec, "Randering Synthetic Objects into Real Scenes : Bridging Traditional and Image Based Graphics with Global Illumination and High Dynamic Range Photography", SIGGRAPH98, pp. 189-198, 1998.

10. Saied Hoezzi et. al, Virtual view generation for 3D digital video, IEEE Multimedia, pp. 18-26, Jan 1997

11. S.E. Chen, L. Williams, "View Interpolation for Image Synthesis", SIGGRAPH93, pp. 279-288, 1993.

12. S.E. Chen, "QuickTime VR-An Image Based Approch to Virtual Environment Navigation", SIGGRAPH95, pp. 29-38, 1995

13. T. Kanade et.al, "Constructing Virtual Words Using Dense Stereo", ICCV98, Bombay, India, pp. 3-10, Jan. 1998.

14. Y. Horry, K.I. Anjyo, K. Arai, "Tour Into the Picture: using a Spidery Mesh Interface to Make Animation from a Single Image", SIGGRAPH 97, pp. 225-232, 1997. 